\title{
LOS TRASTORNOS DE LA PERSONALIDAD DESDE UNA PERSPECTIVA DIMENSIONAL
}

\author{
FERNANDO GAMBA \\ https://orcid.org/0000-0002-8371-6797 \\ MARILINA GRAZIOLA \\ JUAN MARINO \\ MARCOS URGORRI \\ Mariano CASTELLARO \\ Instituto Altué / Rosario, Argentina
}

Recibido: 20 de enero del 2020 / Aceptado: 16 de marzo del 2020 doi: 10.26439/persona2020.n023(1).4830

Resumen. En psicología clínica existe un consenso creciente acerca de las deficiencias del sistema de diagnóstico categorial en el caso de los trastornos de la personalidad (TP), dado que resulta insuficiente para abarcar la experiencia disfuncional del paciente. Esto produce un recorte excesivo y excluye sus aspectos fundamentales e idiosincráticos. Además, impacta negativamente en la conceptualización adecuada del caso y en el diseño de las intervenciones. Cuando existen tantas dificultades para aplicar un modelo diagnóstico a la práctica psicoterapéutica, es posible pensar que lo que debemos modificar es el modelo y no pretender ajustar la psicoterapia a este. Conceptualizar los TP desde la llamada perspectiva dimensional posibilita atender mejor a las particularidades de cada sujeto $y$, de esta manera, incrementar la calidad de las intervenciones. El presente trabajo intenta plasmar cómo dicha perspectiva, que actualmente cuenta con un fuerte soporte empírico, puede mejorar la comprensión clínica de estas perturbaciones y su abordaje terapéutico.

Palabras clave: trastornos de la personalidad / diagnóstico dimensional / diagnóstico categorial

Correo electrónico: lic-juanmarino@hotmail.com 


\section{A DIMENSIONAL APPROACH TO PERSONALITY DISORDERS}

Abstract. In the field of clinical psychology, there is a growing consensus about the deficiencies of the categorical diagnosis system for personality disorders (PD), since it is insufficient to cover the dysfunctional experiences of patients. These deficiencies cause an incomplete system, since it excludes essential and idiosyncratic information necessary to better understand the patients. Moreover, this situation negatively affects the adequate conceptualization of the problems and the design of the interventions. If too many difficulties arise when implementing a diagnostic model in psychotherapy, it is possible to think that the model should be modified instead of trying to adjust psychotherapy to the model. The conceptualization of PD from the so-called "dimensional perspective" makes it possible to address the special features of each subject and, thus, improve the quality of the interventions. This work aims to explain how such dimensional perspective, which currently has a strong empirical support, can improve the clinical understanding of these disorders and their therapeutic intervention.

Keywords: personality disorders / dimensional diagnosis / categorical diagnosis 


\section{INTRODUCCIÓN}

El constructo personalidad ha sido definido de diversas maneras dentro de la literatura. Siguiendo a Belloch Fuster y Fernández Álvarez (2010), la entendemos como una propiedad emergente compleja, que se halla en cambio y crecimiento continuo, en contraposición a una postura tradicional que la concibe en términos de una sumatoria de rasgos (Widakowich, 2012). Más allá de este carácter creciente y dinámico, a lo cual se suma la posibilidad de flexibilizarse para poder hacer frente a las diferentes demandas situacionales o de desarrollo, la personalidad tiende a permanecer relativamente estable a lo largo de la vida, una vez que el individuo alcanza la adultez (Prochaska y Prochaska, 2001). Sus elementos básicos se conceptualizan en términos dimensionales, es decir, los pensamos según un continuo que va desde la presencia mínima o nula de un atributo hasta su máxima expresión, donde lo distintivo de cada sujeto estaría dado por el grado en que dicho atributo se presenta (Hernández-Guzmán, Del Palacio, Freyre y AlcázarOlán, 2011; Mellor Marsá y Aragona, 2009).

En consecuencia, al hablar de la personalidad de un individuo particular nos estamos refiriendo al modo en que habitualmente se comporta, piensa, siente, etcétera. Esto podría alterarse por determinadas circunstancias, por ejemplo, si el sujeto desarrollase un trastorno de pánico con agorafobia, en el cual se vería modificado su modo habitual de sentir y actuar. Sin embargo, a pesar de ello, no podríamos afirmar que se modifique su modo de ser característico y estable. Más bien pensamos en la presencia de un fenómeno disfuncional de carácter transitorio, dado lo cual el sujeto luego retornará a su estilo particular y habitual de ser y comportarse.

En el caso de los trastornos de la personalidad (en adelante, TP), la disfunción se asociaría, juntamente, al modo de ser, estable, omnipresente y egosintónico (Belloch Fuster y Fernández Álvarez, 2010). El individuo sufre y se ve imposibilitado de desarrollarse plenamente. Sin embargo, no vincula sus dificultades diarias con su modo de ser, al cual se le pueden atribuir, además, las características de rígido e inflexible. Con esto último queremos indicar que se vería significativamente afectada la posibilidad de modificar determinadas creencias y conductas para poder hacer frente a circunstancias ambientales estresantes. Las áreas en que se manifiestan los trastornos de la personalidad son la cognición (forma de percibir e interpretarse a sí mismo, a los otros y a los sucesos), el área afectiva (rango, intensidad, labilidad y adecuación de la respuesta emocional), el control de los impulsos y la necesidad de gratificación, así como el modo de relacionarse con los demás.

Las personas con un TP son especialmente vulnerables y frágiles en las situaciones de estrés, que les demandan otras estrategias, además de las habituales, para lograr reducir la tensión o malestar. Se trata de un fenómeno complejo que requiere de un abordaje terapéutico también complejo. Para poder diseñarlo, necesitamos un modelo 
psicopatológico acorde con dicha característica (Bowins, 2010; Um, Hershberger, Whitt y Cyders, 2018).

\section{EL PROBLEMA DE LA CLASIFICACIÓN}

Tradicionalmente, los TP han sido definidos según un esquema categorial, en el eje Il del Manual diagnóstico y estadístico de los trastornos mentales (DSM). En su cuarta versión (American Psychiatric Association, 1995), se describen doce trastornos, a saber, paranoide, esquizoide, esquizotípico (agrupados en la categoría denominada "extraños y excéntricos"), antisocial, límite, histriónico, narcisista (agrupados como los "dramáticos, emocionales e impulsivos"), evitativo, dependiente, obsesivo-compulsivo (denominados los "ansiosos y temerosos"), pasivo-agresivo, depresivo (en el grupo "trastornos de la personalidad en estudio") y la categoría residual "trastorno de la personalidad no especificado". Posteriormente, la quinta versión del DSM (American Psychiatric Association, 2013) retomó las diez primeras formas de TP. Para el diagnóstico se requiere la presencia de un patrón inflexible y persistente de experiencia interna y conducta, que se desvía notablemente de las expectativas culturales, se inicia en la adolescencia o al comienzo de la adultez y se manifiesta en al menos dos de estas áreas: cognición, afectividad, funcionamiento interpersonal y control de impulsos; produce deterioro social o laboral, y no se explica mejor por la presencia de otro trastorno mental ni se debe a los efectos de alguna sustancia o condición médica general.

Bajo las formas de presentación antes descritas (y la posibilidad de comorbilidad), los psicólogos clínicos nos acostumbramos durante mucho tiempo a pensar estas disfunciones en el modo de ser que llamamos TP. Sin embargo, desde hace algunos años, comenzamos a tomar conciencia de que dicha clasificación puede no ser la adecuada (Calvo et al., 2016; Esbec y Echeburúa, 2014). Más aún, poco aportaría al proceso psicoterapéutico del paciente (Beutler, Someah, Kimpara y Miller, 2016).

Los terapeutas necesariamente tenemos un modelo desde el cual intervenimos y desde el cual, primero, decodificamos lo que el paciente nos relata. Y es quizá inevitable que amoldemos su experiencia a nuestras categorías mentales, derivadas del modelo con el cual trabajamos. El problema aparece cuando este modelo produce un recorte tan grande que ocasiona una pérdida significativa de información, que hubiese sido relevante para entender mejor la experiencia del paciente $y$, con base en ese entendimiento, diseñar una mejor estrategia terapéutica para poder ayudarlo.

Si pensamos en un encuentro terapéutico, tenemos, por un lado, la experiencia -intransferible - del paciente (que proviene de su mundo interno) y, por otro, la representación de esta que se hace el terapeuta. Así, el acercamiento estaría dado por el nivel de empatía que pueda lograr el terapeuta respecto de la experiencia de quien consulta y las categorías clínicas de las que disponga para conceptualizar el caso. 
Al intervenir es importante movernos en el mismo universo de significados de nuestro interlocutor. No redundar en la misma forma de narrar la historia, pero sí procurar acceder lo más posible al modo en que lo hace y ayudarlo a relatar de un modo diferente. Si bien nunca podemos saber con exactitud cuál será el impacto de una intervención, mientras más refinada sea nuestra comprensión de la experiencia interna del paciente (basada en un modelo mental suficientemente abarcativo), más oportunidades tendremos de que dicha intervención surta el efecto deseado.

\section{Dificultades de los modelos categoriales}

Hasta el momento no se tienen pruebas de que la utilización del concepto de trastorno de la personalidad, entendido en sentido categorial, haya hecho grandes aportes para la clínica psicoterapéutica. Es más, creemos que ha agregado una complicación, dado que constituye un constructo teórico limitante. Para el establecimiento del diagnóstico de TP, hay consenso en que lo fundamental es (hasta el momento) el juicio clínico (Belloch Fuster y Fernández Álvarez, 2010). La esperanza se centraría en la construcción de métodos diagnósticos más eficaces que los existentes, para dar una definición más precisa acerca de cuándo estamos en presencia de una disfunción en el modo de ser y cuándo no.

Un segundo problema es la coocurrencia entre los diferentes TP (Widiger, 2003). Las diferentes categorías se solapan entre sí y, en la práctica, es poco frecuente encontrar un TP puro; por eso, se diagnostica que los sujetos presentan varios trastornos de la personalidad a la vez (dado que ninguno, considerando su descripción individual, logra abarcar la complejidad de la disfunción).

Un tercer problema es la alta coocurrencia de los TP con los trastornos del eje I (Widiger, 2003), lo que a veces ocasiona que los primeros sean obviados en el diagnóstico o confundidos con el cuadro sintomático. Por ejemplo, se diagnostica un cuadro de ansiedad sin contemplar la presencia de un trastorno evitativo de la personalidad; esto tendría un impacto tanto en la remisión de los síntomas (dificultando el cambio terapéutico en el corto plazo) como en la probabilidad de recaídas.

Quizá la principal dificultad para establecer criterios útiles para la clínica es pretender trabajar con los TP sin definir adecuadamente el constructo personalidad. Además, dado que es difícil establecer el punto de corte entre TP y no TP, a la hora de intervenir posiblemente resulte más útil basarse en variables clínicas continuas y pensar cuándo, dónde y hasta dónde realizar la intervención.

Lo que necesitamos, entonces, es construir mejores modelos del funcionamiento mental para la comprensión de la experiencia del paciente. Una posible vía de solución podrían ser los modelos dimensionales de los trastornos de la personalidad (Calvo, Mata, 
Pérez Sayes y Tirapu Ustárroz, 2005; O'Connor, 2005; Reynolds y Clark, 2001; Simonsen y Widiger, 2005). La última versión del DSM contempla una perspectiva dimensional para la conceptualización de los TP, aunque también conserva el modelo tradicional, en un intento por mantener la continuidad con la práctica clínica clásica e introducir una nueva aproximación (Witkiewitz et al., 2013). Sin embargo, la quinta versión ha mostrado escasa utilidad desde su publicación (Artigas-Pallarés y Paula-Pérez, 2015), y no solo en el terreno de los trastornos de la personalidad. Quizá la propuesta superadora para los clínicos en cuanto a la conceptualización de estos trastornos deba buscarse fuera del sistema DSM.

\section{La alternativa dimensional}

Widiger y Lowe (2007) proponen una reseña de las principales propuestas de alternativas para una clasificación dimensional de los TP. Estas son las siguientes: una clasificación dimensional de las categorías existentes, las subescalas del Dimensional Assessment of Personality Pathology (DAPP; Livesley y Jackson, 2009) y/o del Schedule for Nonadaptative and Adaptative Personality (SNAP; Clark, 1993), las tres polaridades de Millon (Millon y Grossman, 2006), el modelo de los siete factores de Cloninger (1999) y el modelo de los cinco factores (FFM) de McCrae y Costa (1996). De estos modelos, el que ha recibido mayor respaldo empírico es el último.

El FFM postula cinco dimensiones fundamentales de la personalidad: amabilidad, responsabilidad (o escrupulosidad o restricción), extraversión (o afectividad positiva), neuroticismo (o afectividad negativa) y apertura a la experiencia (o falta de convencionalismo). Cada dimensión incluye un polo negativo: hostilidad o ira (opuesto a amabilidad), negligencia o irresponsabilidad (opuesto a responsabilidad), introversión (opuesto a extraversión), estabilidad emocional (opuesto a neuroticismo) y convencionalismo o cerrazón (opuesto a apertura a la experiencia). Además, el modelo considera factores de segundo orden (llamados facetas), englobados dentro de cada uno de los cinco grandes factores de primer orden. Tanto estos como sus facetas correspondientes tienen un carácter dimensional, es decir, fluctúan en intensidad a lo largo de un continuo. El significado y las consecuencias de cada atributo estarían dados por la ubicación dentro de este continuo.

Si nos posicionamos desde esta perspectiva, los trastornos de la personalidad podrían entenderse como variantes patológicas de los dominios y las facetas de la personalidad normal (Fernández Álvarez, Consoli y Gómez, 2016). En otras palabras, en un modelo de estas características, se considera que los trastornos mentales no son, en realidad, cualitativamente diferentes de la normalidad. Los elementos característicos de las disfunciones serían los mismos de la personalidad normal, pero bajo formas extremas (por exceso o por defecto), y lo que daría lugar a una personalidad patológica 
sería la combinación de los rasgos/tendencias básicas con la influencia ambiental. Este planteamiento redunda en una conceptualización del cuadro que nos brinda el acceso a mucha mayor información sobre el consultante, en comparación con aquella derivada de la descripción contenida en los criterios diagnósticos según un modelo categorial. No se buscará ya definir las posibles variantes de un TP, sino conocer cuáles son los rasgos específicos (y la intensidad con que se manifiestan) que caracterizan la patología de personalidad de un paciente dado. Sobre esta base, se puede realizar un diseño de intervención mucho más ajustado a las necesidades de cada sujeto.

En el artículo antes mencionado, Widiger y Lowe (2007) plantean también que los modelos dimensionales se podrían integrar en una estructura jerárquica común. Según los autores, el FFM puede, por ejemplo, integrarse bien con el modelo DAPP y con el modelo SNAP (estos últimos ya en el terreno de las disfunciones de personalidad). Hasta el momento no contamos con un modelo dimensional integrado, basado en una teoría de la personalidad y sus disfunciones, que pueda dar respuesta a las múltiples dificultades que se nos presentan en la clínica. A pesar de ello, este intento por conceptualizar mejor la experiencia de sufrimiento humano produce ya un efecto en el terreno práctico, que consiste en modificar la forma en que escuchamos a nuestros pacientes. Además, contamos con la ayuda de diferentes instrumentos para la tarea diagnóstica: algunos son cuestionarios e inventarios y otros son entrevistas estructuradas. El Inventario de Personalidad Neo Revisado (NEO PI-R, de McCrae y Costa, 2002) y la Structured Interview for the Five-Factor Model of Personality (SIFFM, de Trull y Widiger, 1997) son ejemplos de ello. Utilizar estos recursos resulta sin duda beneficioso y ahorra tiempo. Por otra parte, sirve a los fines de controlar los posibles sesgos de una evaluación realizada exclusivamente a través del juicio clínico.

Estamos avanzando en el intento por construir un sistema de diagnóstico más eficiente. En el futuro, quizá este cambio dé lugar también a un mayor consenso, en vías de consolidar un modelo dimensional unificado.

\section{CONCLUSIÓN}

El sistema de clasificación categorial para los trastornos de la personalidad resulta escasamente útil; es claro que se trata de un modelo en crisis, ya desde hace tiempo. Cuando existen tantas dificultades para aplicar un modelo a la práctica psicoterapéutica, es posible pensar que lo que tenemos que modificar es el modelo y no pretender ajustar la psicoterapia a este. Resulta interesante, además, que la mayoría de las categorías diagnósticas tampoco ayuden a establecer el pronóstico o la línea de tratamiento. Por otra parte, dejan fuera aspectos fundamentales del sujeto que nos consulta, es decir, sus particularidades y cualidades distintivas e idiosincráticas. 
Los terapeutas tenemos ahora frente a nosotros la posibilidad de operar con un nuevo criterio, que, por otra parte, cuenta con un fuerte soporte empírico. Conceptualizar los trastornos de la personalidad desde una perspectiva dimensional posibilita atender mejor a las particularidades de cada sujeto y así mejorar la calidad de las intervenciones que realizamos.

\section{REFERENCIAS}

American Psychiatric Association. (1995). Diagnostic and Statistical Manual of Mental Disorders: DSM-IV. Washington D. C.: Autor.

American Psychiatric Association (2013). Diagnostic and Statistical Manual of Mental Disorders: DSM-V. Washington D. C.: Autor.

Artigas-Pallarés, J., y Paula-Pérez, I. (2015). Asignaturas pendientes del DSM-5. Revista de Neurología, 60(1), 95-101.

Belloch Fuster, A., y Fernández Álvarez, H. (2010). Tratado de trastornos de la personalidad. Madrid: Síntesis.

Beutler, L., Someah, K., Kimpara, S., y Miller, K. (2016). Selecting the most appropriate treatment for each patient. International Journal of Clinical and Health Psychology, 16(1), 99-108.

Bowins, B. (2010). Personality disorders: a dimensional defense mechanism approach. American Journal of Psychotherapy, 64(2), 153-169.

Calvo, R., Mata, I., Pérez Sayes, A., y Tirapu Ustárroz, J. (2005). Propuesta de un modelo dimensional para los trastornos de personalidad. Actas Españolas de Psiquiatría, 33(4), 254-262.

Calvo, N., Valero, S., Sáez-Francàsc, N., Gutiérrez, F., Casas, M., y Ferrera, M. (2016). Borderline personality disorder and personality inventory for DSM-5 (PID-5): dimensional personality assessment with DSM-5. Comprehensive Psychiatry, 70, $105-111$.

Clark, L. A. (1993). Schedule for Nonadaptive and Adaptive Personality (SNAP). Mineápolis, $\mathrm{MN}$ : University of Minnesota Press.

Cloninger, C. R. (1999). Personality and psychopathology. Arlington, VA: American Psychiatric Association.

Esbec, E., y Echeburúa, E. (2014). La evaluación de los trastornos de la personalidad según el DSM-5: recursos y limitaciones. Terapia Psicológica, 32(3), 255-264.

Fernández Álvarez, H., Consoli A. J., y Gómez B. (2016). Integration in psychotherapy: reasons and challenges. American Psychologist, 71(8), 820-830. 
Fernández Álvarez, H., y Fernández Álvarez, J. (2017). Terapia cognitivo conductual integrativa. Revista de Psicopatología y Psicología Clínica, 22, 157-169.

Hernández-Guzmán, L., Del Palacio, A., Freyre, M., y Alcázar-Olán, R. (2011). La perspectiva dimensional de la psicopatología. Revista Mexicana de Psicología, 28(2), 111-120.

Livesley W. J., y Jackson, D. N. (2009). Manual for the Dimensional Assessment of Personality Pathology-Basic Questionnaire. Port Huron, MI: Sigma Press.

McCrae, R. R., y Costa, P. T. (1996). Toward a new generation of personality theories: theoretical contexts for the five-factor model. En J. S. Wiggins (Ed.), The fivefactor model of personality: theoretical perspectives (pp. 51-87). Nueva York, NY: The Guilford Press.

McCrae, R. R., y Costa, P. T. (2002 [1978]). Inventario de Personalidad Neo Revisado (NEO $P I-R)$. Madrid: TEA Ediciones.

Mellor Marsá, B., y Aragona, M. (2009). De la categoría a la dimensión: una mirada crítica a la evolución de la nosografía psiquiátrica. Revista de la Asociación Española de Neuropsiquiatría, 29(103), 217-228.

Millon, T., y Grossman, S. D. (2006). Goals of a theory of personality. En J. C. Thomas, D. L. Segal y M. Hersen (Eds.), Comprehensive handbook of personality and psychopathology, Vol. 1: Personality and everyday functioning (pp. 3-22). Hoboken, $\mathrm{NJ}$ : John Wiley \& Sons Inc.

O'Connor, B. (2005). A search for consensus on the dimensional structure of personality disorders. Journal of Clinical Psychology, 61(3), 323-345.

Prochaska, J. M., y Prochaska, J. O. (2001). ¿Por qué no se mueven los continentes? ¿Por qué no cambian las personas? Revista de Psicoterapia, 12(46-47), 17-36.

Reynolds, S., y Clark, A. (2001). Predicting dimensions of personality disorder from domains and facets of the five-factor model. Journal of Personality, 69(2), 199-222.

Simonsen, E., y Widiger, T. (2005). Alternative dimensional models of personality disorder. Journal of Personality Disorders, 19(2), 110-130.

Trull, T. J., y Widiger, T. A. (1997). Structured Interview for the Five-Factor Model of Personality. Odessa, FL: Psychological Assessment Resources.

Um, M., Hershberger, A., Whitt, Z., y Cyders, M. (2018). Recommendations for applying multi-dimensional model of impulsive personality to diagnosis and treatment. Borderline Personality Disorder and Emotion Dysregulation, 5(6), 1-17.

Widakowich, C. (2012). El enfoque dimensional vs. el enfoque categórico en psiquiatría: aspectos históricos y epistemológicos. Alcmeón, Revista Argentina de Clínica Neuropsiquiátrica, 17(4), 365-374. 
Widiger, T. (2003). Personality disorder diagnosis. World Psychiatry, 2(3), 131-135.

Widiger, T. A., y Lowe, J. R. (2007). Five-factor model assessment of personality disorder. Journal of Personality Assessment, 89(1), 16-29. https://doi. org/10.1080/0022 3890701356953

Witkiewitz, K., King, K., McMahon, R., Wu, J., Luk, J., Bierman, K., Coie, K., Dodge, K., Greenberg, M., Lochman, J., y Pinderhughes, E. (2013). Evidence for a multidimensional latent structural model of externalizing disorders. Journal of Abnormal Child Psychology, 41, 223-237. 